\title{
Ethics and Prevention of Chronic Disease
}

\section{Hoffman DP*, Robitscher J and Mertzlufft J}

Department of Ethics and Health Policy, Maria College, USA

*Corresponding author: David P Hoffman, Associate Dean for Academic Initiatives and Government Affairs, Department of Ethics and Health Policy, Maria College 700 New Scotland Ave, Albany, New York 12208, USA, Tel: 5183667544; Email: dhoffman@mariacollege.edu
Short Communication

Volume 4 Issue 1

Received Date: February 05, 2021

Published Date: February 19, 2021

DOI: $10.23880 /$ abca-16000161
Abbreviations: DPP: Diabetes Prevention Program; DSMES: Diabetes Self-Management Education and Support; FQHCs: federally qualified health centers; CDC: chronic disease prevention and control.

\section{Short Communication}

We hear much discussion about healthcare coverage and costs associated with insurance, pharmaceuticals, surgical interventions, and related issues. All of these are important but leave out perhaps the most important strategy prevention.

\section{Facts We Must Acknowledge}

The urgency of addressing chronic disease can't be stressed enough these conditions account for over $86 \%$ of our healthcare costs, and much of this is preventable $[1,2]$. This literally means that if invested in properly, we could spend $\$ 240$ now on prevention instead of $\$ 1000$ in the future on reactive healthcare costs for chronic disease. Healthcare costs are only the tip of the iceberg. Absenteeism (time taken off due to illness or other reasons) and presenteeism (attending despite an illness that prevents full functioning) in school and at work take a significant toll on family life, the ability to plan for their future, and our global economic competitiveness [3].

Almost every American family is adversely affected by chronic diseases in one way or another through the death of a loved one or family members with life-long illness, disability, or compromised quality of life. These burdens affect society on both the personal and community level, not just in the physical disease, but also in the financial burden that comes with the cost of chronic disease [4].

We are beginning to see the impact of the Covid 19 pandemic on the population. This burden isn't carried equally, black and Hispanic people, seniors, and other groups carry a disproportionate share, very much the same as many chronic conditions. This is further evidence that health risk for these groups including people in poverty outweighs many factors previously credited with differences.

At a time when our investments in housing, education, and medical care have outstripped inflation, our investment in prevention has lagged far behind. Today we fund prevention efforts at approximately the same amount we did in 2001 , effectively a funding cut of $22.92 \%$ when inflation is considered.

To summarize and focus let's consider a reality check:

- As of 2014,60\% of American adults had at least one chronic condition, and $42 \%$ had more than one chronic condition.

- Chronic diseases such as heart disease, cancer, and diabetes are the leading causes of death and disability in the United States. 7 in 10 leading causes of death in 2017 were chronic diseases, totaling about 1.75 million American deaths.

- More than $86 \%$ of the nation's healthcare costs relate to chronic diseases, and most of those costs are preventable [5-8].

- The projected prevalence of any cardiovascular disease in the United States will increase by $3 \%$ to over $45 \%$ by the year 2035 [9].

- Risk factors such as poor diet, lack of activity, alcohol abuse, and ignoring medical advice all contribute too much to this crisis.

- $27 \%$ of young adults are too overweight to serve in the military [10].

\section{Change is Overdue}

We have created a culture where the healthy choice is often the hardest choice at every stage of our lives. We know 
we need to eat better, be more active and avoid tobacco - but we're cutting back on recess and physical education, cutting back on the ability to be active in our everyday lives and tobacco is still all too available, especially to children [1115]. The CDC estimates that modifying three risk factors poor diet, lack of physical activity, and smoking can prevent $80 \%$ of heart disease and stroke, $80 \%$ of type 2 diabetes, and $40 \%$ of cancer. Good, healthy food options are more available today but not everywhere and not for everyone. If we are serious about improving the lives of Americans, having an impact on healthcare costs, reforming our system, and reducing disparities, we need to invest in a meaningful way in prevention $[16,17]$.

\section{We can Bring Chronic Disease Prevention Up to Scale}

Today, only a fraction of $1 \%$ of federal healthcare investment goes to prevention this is a breach of an ethical approach to public policy, especially since we know better. Even with this limited funding, states are implementing diverse, cost-effective strategies that work for early detection of cancer, prevention and control of diabetes, reduction of heart disease and stroke, reduction of the disability associated with all these conditions, and beginning key programs targeting arthritis and Alzheimer's disease using the latest science. To continue this work, there needs to be a substantial investment in CDC's National Center for Chronic Disease Prevention and Health Promotion to allow them to assure that every state and territory has a full complement of programs addressing these challenges to our health.

State Chronic Disease Directors and the State Health Agencies where they work have a unique role in coordinating activity and steering resources to communities most in need and creating linkages across systems with healthcare providers, insurers, educators, community organizations, and others. States effectively maximize federal investments and ensure the most efficient mobilization of local organizations, while at the same time avoiding any duplication.

The minimal investment in chronic disease prevention and control at CDC, state and community-based programs supported through CDC, and states has resulted in developing an extensive portfolio of strategies that work. With current financial resources these programs are not scalable across the nation. This is the largest barrier we are facing in regards to preventing expensive chronic diseases.

The federal investment needs to be such that every state has a cadre of evidence based programs to fight chronic disease including:

- Early Detection of Cancer and Cancer Survivorship Services
- Diabetes Prevention and Control (including prevention of related kidney disease)

- Heart Disease and Stroke Prevention

- Healthy Community Programs (REACH, others)

- Tobacco Prevention and Control

- Alzheimer's Disease Program (Healthy Brain Initiative)

- Arthritis Prevention and Control

- School Health and Oral Health Programs

- Post-Covid19 Chronic Conditions

\section{Public Health Chronic Disease Prevention Programs Save Lives and Money some examples:}

\section{Obesity}

Illinois: The Illinois Department of Public Health worked with five Illinois counties in suburban Chicago to improve healthy eating behaviors through implementation and adoption of food service guidelines at worksites. The tailored nutrition standards impacted over 1800 local health department employees and community members attending local health department-sponsored events.

Pennsylvania: The Pennsylvania Department of Health partnered with The Food Trust to train community partners how to work with store owners to stock and promote healthier food and beverages. As a result, over 150 stores serving 890,000 residents in 10 of the most populated cities in Pennsylvania now offer healthier food and beverage options.

\section{Diabetes}

Kentucky: From 2013-2018, the Kentucky Department for Public Health expanded the National Diabetes Prevention Program (DPP) by:

- Increasing the number of CDC-recognized program delivery organizations in the state from 2 to 54 ;

- Increasing geographic coverage from two metropolitan counties to an additional 86 rural counties;

- Increasing access to in-person programs for $87 \%$ of the population compared to only $24 \%$ in 2012 ; and

- Increasing the number of employers offering the National DPP lifestyle change program as a covered health benefit for their employees from 0 to 33.

All public employees and adult dependents are now covered. Montana: The Eastern Montana Telemedicine Network launched a Diabetes Self-Management Education and Support (DSMES) initiative that included a telehealth component in five rural clinics where certified diabetes educators served on a team of health professionals to deliver both DSMES and clinical care. Amongst participants, the practice of checking blood glucose correctly for 6 months improved from 31\% at baseline to $61 \%$ after the intervention and healthy diet 
improved from $8 \%$ to $29 \%$. Additionally, $97 \%$ of the group stated that telehealth was a useful tool for delivering DSMES. The number of DSMES sites across the state increased to 36, with 10 programs offering a telehealth option.

\section{Heart Disease and Stroke}

Michigan: Michigan has implemented systems and practices to improve care and enhance prevention of risk factors like high blood pressure for tens of thousands of people. The Michigan Department of Health and Human Services) partnered with a primary care association and others to improve the quality of heart care. In four years the state reported:

- All patients served by federally qualified health centers (FQHCs) are covered by electronic health records that include criteria for treating and improving care of high blood pressure.

- $87 \%$ of $\mathrm{FQHCs}$ were covered by policies promoting team-based care to treat high blood pressure, up from $40 \%$ in just 4 years.

- A project targeting the African-American community in Muskegon Heights saw 2,500 clients initiate a blood pressure self-management plan and referred nearly 4,000 adults with high blood pressure to community resources for treatment.

Wisconsin: The Wisconsin Primary Healthcare Association partnered with the Chronic Disease Quality Improvement Project and the Wisconsin Collaborative for Healthcare Quality to implement new health information technology systems that improve workflows for identification and care of patients with undiagnosed high blood pressure. These three key partners saw a collective $6 \%$ increase in patients with controlled blood pressure from 2013-2018. Additional CDC-supported activities have generated significant benefits for Wisconsin residents.

- In $2017,94.5 \%$ of reporting healthcare systems used electronic health records appropriate for treating high blood pressure. This represents nearly a $10.4 \%$ increase during the course of the project and surpasses the prior benchmark.

- By $2017,71 \%$ of health systems had policies to encourage multidisciplinary team-based approaches, up from $52 \%$ earlier.

\section{Cancer}

West Virginia: Wheeling Health Right raised its colorectal cancer screening rate by 58 percentage points in two years. The CDC-funded West Virginia Program to Increase Colorectal Cancer Screening made the following changes:

- Changing to a more patient-friendly screening method,

- Reviewing and updating patient charts with screening data, and
- Reaching out with patient reminders. These changes raised the clinic's screening rate from $9 \%$ to $53 \%$ in 2017, and then to more than $67 \%$ in 2018.

New York: New York State provided administrative support and tailored education on quality improvement, cancer screening, and data improvement to eight primary care practices serving low-income, uninsured, racial and ethnic minority, and geographically isolated women. This was done to address system-level barriers to increase breast, cervical, and colorectal cancer screening rates. After 5 years, the practices increased their screening rates by more than $10 \%$. The most successful practices were those that implemented patient and provider reminder systems, policy changes, pre-office visit planning, provider education, and mobile mammography van services.

\section{Alzheimer's}

Colorado: To develop a dementia-competent workforce, the Colorado Department of Health and the Environment partnered with the Alzheimer's Association Colorado Chapter to deliver its Approaching Alzheimer's: First Responder Training Program. The free in-person program reached all 11 of Colorado's Regional Emergency and Trauma Advisory Councils. The training helps first responders serve people with Alzheimer's in situations involving wandering, disasters or other emergency situations, abuse or neglect, "shoplifting" because they forgot to pay, and driving.

Alzheimer's disease and Healthy Aging Program, Utah: A collaboration involving five Utah health organizations one of which surveyed Utah's healthcare providers resulted in two validated cognitive assessment tools physicians are currently using during Medicare wellness visits. The assessment tools are the Mini-Cog, followed by the Montreal Cognitive Assessment tool. The Utah project deepened the understanding of some challenges physicians face in assessing cognition, one of which is uncertainty about which validated tool to use.

\section{The Ethical Dilemma}

America is on the precipice of great challenges and great opportunities; we need to invest in a meaningful way in prevention now before it is too late, and we are too ill to invest. The place to start is at CDC, with the statebased programs reaching localities across the country as mentioned above. The bioethics principle of justice calls for fairness, resource allocations are currently anything but fair. The bioethics principle of autonomy calls for respecting the informed decisions of individuals. These decisions cannot be fully informed without public health programs to educate populations (especially those at greatest risk). The ethical principle of beneficence calls us to "do good". We know that these strategies can make a difference we need to 
support them to be beneficent. Finally, the principle of nonmaleficence more commonly known as "do no harm" requires that we act on our knowledge to avoid the predictable harm that is occurring and will only increase without these investments.

In case there is remaining doubt, Trust for America's Health reported the return on investment for public health prevention programs is 0.96:1 in years one and two and 5.6:1 by year five. This means that even in the initial budget period there would be a net gain by investing in prevention.

Public health programs improve care, prevent disease, and prevent complications of disease. An investment in chronic disease prevention and control programs saves lives, improves quality of life, and saves healthcare dollars.

\section{References}

1. Schneider EC, Sarnak DO, Squires D, Shah A, Michelle M (2017) Doty International Comparison Reflects Flaws and Opportunities for Better U.S. Health Care. The Commonwealth Fund.

2. (2017) Health expenditures. Centers for Disease Control and Prevention website.

3. (2017) NHE fact sheet. Centers for Medicare and Medicaid Services.

4. (2016) Health insurance coverage in the United States. United States Census Bureau.

5. (2017) Chronic Disease. Centers for Disease Control and Prevention website.

6. (2011) Chronic disease prevention. Centers for Disease Control and Prevention website.

7. Fullman N, Barber RM, Abajobir AA (2017) Measuring progress and projecting attainment on the basis of past trends of the health-related sustainable development goals in 188 countries: an analysis from the global burden of disease study 2016. Lancet 390(10100): 1423-1459.

8. Khavjou O, Phelps D, Leib A (2016) Projections of cardiovascular disease prevalence and costs: 20152035. RTI International.

9. (2017) Facts and statistics. U.S. Department of Health \& Human Services.

10. (2017) CDC's Work to Reduce Childhood Obesity by Connecting Families, Clinics, and Communities. Centers for Disease Control and Prevention.

11. (2018) Adolescent and School Health. Centers for Disease Control and Prevention.

12. (2018) US Opioid Crisis Addressing Maternal and Infant Health. Centers for Disease Control and Prevention, pp: 1-2.

13. Zzzzzz...Sleepy Kids- Most Students Need More Sleep. Centers for Disease Control and Prevention.

14. (2018) Talk with Your Teen About E-cigarettes: A Tip Sheet for Parents. Know the Risks E-Cigarettes \& Young People.

15. (2018) Members and Veterans of the Armed Forces. Centers for Disease Control and Prevention.

16. (2018) Making Alzheimer's Our Next Public Health Success Story. Centers for Disease Control and Prevention.

17. Hoffman D (2019) Public Health Responding to the Epidemic of Alzheimer's disease. Ageing Sci Ment Health Stud 3(3): 1-2.

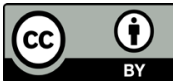

